\title{
TERATOMA CERVICAL FETAL GIGANTE: A PROPÓSITO DE UN CASO
}

\author{
Jaime Ingar Pinedo", Erasmo Huertas Tacchino ${ }^{1}$, Oscar Antonio Limay Ríos ${ }^{1}$, Walter Castillo Urquiaga ${ }^{1}$, Mario Zarate \\ Girao', José Quispe Zuñiga ${ }^{2}$, Elizabeth Betteta Espejo ${ }^{3}$
}

\begin{abstract}
RESUMEN
Los tumores fetales cervicales son hallazgos infrecuentes y la mayor parte de los casos no son diagnosticados prenatalmente. El objetivo de este trabajo es presentar y discutir en forma crítica el diagnóstico, evaluación y evolución perinatal de un caso de un tumor cervical fetal gigante, así como su diagnóstico diferencial.
\end{abstract}

Palabras clave: Teratoma; Fetal; Tumor cervical (fuente: DeCS BIREME).

\section{FETAL CERVICAL TERATOMA GIANT: A CASE REPORT}

\begin{abstract}
Fetal cervical tumors are infrequent findings and most of them are not diagnosed prenatally. The objective of this paper is to present and discuss in a critical way the diagnosis, evaluation and post natal evolution of a giant fetal cervical tumor case, also of his differential diagnosis.
\end{abstract}

Key words: Teratoma; Fetal; Cervical tumor (source: MeSH NLM).

\section{INTRODUCCIÓN}

Los teratomas cervico faciales en los recién nacidos comprenden entre el 1,5 al $5,5 \%$ de los teratomas en general y tienen una incidencia de 1:20,000 a 1:80,000 nacidos vivos. Los teratomas cervicales son raros y sólo se encuentran reportados como casos únicos o series de pocos pacientes ${ }^{1}$; usualmente afectan a ambos sexos y a todas las razas en igual proporción ${ }^{2}$. Estos tumores suelen presentarse de forma aislada, sin asociación con otras anomalías cromosómicas o malformaciones congénitas, pero algunos autores afirman que al ser un grupo heterogéneo de tumores hay varios genes implicados en su proceso patogénico ${ }^{3}$. En general los teratomas están constituidos por las 3 líneas germinales: endodermo, mesodermo y ectodermo y representan el 25 al $35 \%$ de todos los tumores fetales ${ }^{4}$.

Los teratomas cervicales son masas bien delimitadas y voluminosas, con componentes sólidos y quísticos, que miden de 5 a $12 \mathrm{~cm}$ en su diámetro mayor, pudiendo ser maduros e inmaduros. A pesar de que son benignosen95\% de los casos el teratoma cervical no tratado puede conducir a la muerte en aproximadamente el $80 \%$ de los casos, debido principalmente a la enfermedad respiratoria. Las consecuencias del tumor sobre el neonato se determinan por el tamaño, ubicación, tasa de crecimiento y poder infiltrativo de la lesión, pudiendo llegar a ser mortal en algunos $\operatorname{casos}^{1,2}$.
El objetivo de este reporte de caso es brindar información acerca de esta patología, debido a los pocos datos existentes y al mínimo conocimiento acerca de teratomas congénitos en nuestro medio; así como aportar bases clínicas para el enfoque diagnóstico y manejo al personal de salud que en su práctica habitual tenga contacto con recién nacidos.

\section{REPORTE DE CASO}

Paciente mujer de 24 años, procedente de Loreto, Gestante de 38 semanas, acude para ecografía obstétrica doppler con diagnóstico presuntivo de higroma quístico, el cual fue realizado por una ecografía particular. Paciente tiene como antecedentes de importancia una cesárea anterior en el 2006 por distocia funicular y 02 legrados por aborto incompleto de 7 y 8 semanas respectivamente; niega otros antecedentes. No tiene controles prenatales. Se realiza ecografía obstétrica en la unidad de medicina fetal del Instituto Nacional Materno Perinatal cuyo diagnóstico son: gestación de 38 semanas por biometría fetal, doppler de arteria umbilical normal (IP: 0,9), líquido amniótico adecuado (ILA: $8 \mathrm{~cm}$ ) y una tumoración cervical fetal gigante a predominio izquierdo de medidas $96 \times 101 \mathrm{~mm}$ de diámetro de contenido heterogéneo con imágenes anecoicas internas con tabiques delgados con poca vascularización central al doppler color, se concluye como probable linfoadenoma cervical fetal (Figura 1).

Médico Gineco Obstetra de la Unidad de Medicina Fetal del Instituto Nacional Materno Perinatal. Lima Perú

Médico Residente de Gineco-Obstetricia del Instituto Nacional Materno Perinatal (Ex Maternidad de Lima). Lima Perú.

3 Médico Residente de Gineco-Obstetricia del Hospital Nacional Dos de Mayo. Lima-Perú.

Recibido: 10-10-12 Aprobado: 22-04-13 


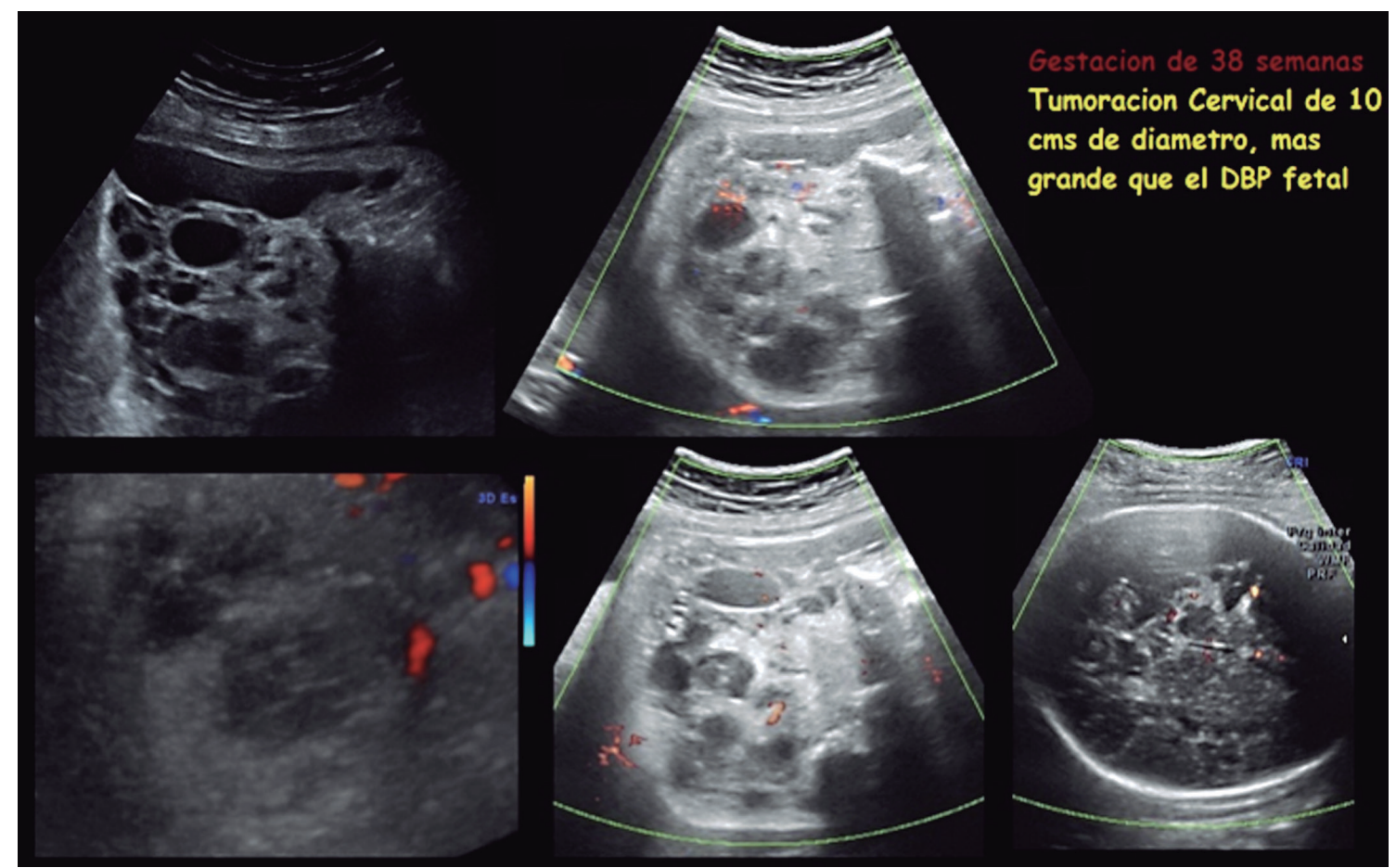

Figura 1. Ecografía medicina fetal con tumoración cervical fetal gigante.

Paciente cursa con dinámica uterina regular durante la evaluación ecográfica por lo que es remitida al servicio de emergencia para evaluación. El mismo día es evaluada y sometida a cesárea de emergencia por no condiciones para parto vaginal por la presencia de la tumoración cervical. Se termina la cesárea sin complicaciones.

Se obtiene un recién nacido varón, de 37 semanas por método Capurro; peso: 3530 g; Apgar 8 al minuto y 9 a los 5 minutos; con tumoración cervical de $12 \times 6$ centímetros que compromete región inframandibular. Recién nacido pasa a cuidados intermedios donde desarrolla dificultad respiratoria progresiva de tipo obstructivo, recibiendo intubación endotraqueal, no presentando mejoría y falleciendo a los 02 días de nacido.

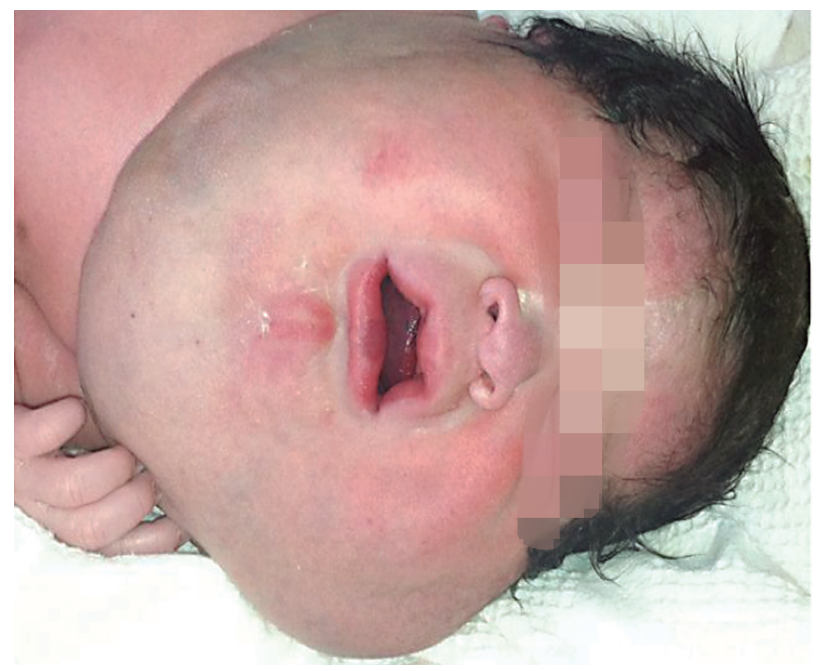

\section{DISCUSIÓN}

Los tumores cervicales fetales son lesiones muy raras y su diagnóstico diferencial también incluye los teratomas; el diagnóstico prenatal ha sido escasamente reportado en la literatura ${ }^{5}$.

Aunque su apariencia sonográfica no puede ser usada para diferenciarla de otra patología, la localización es muy importante, siendo más frecuente a nivel cervical anterior. La apariencia típica es el de una masa ecogénica con áreas hipoecogénicas, éstos tienen un componente sólido más heterogéneo, conteniendo quistes y calcificaciones ${ }^{11}$. Se recomienda el estudio doppler para definir los bordes de la lesión, el cual debió realizarse como estudio en el

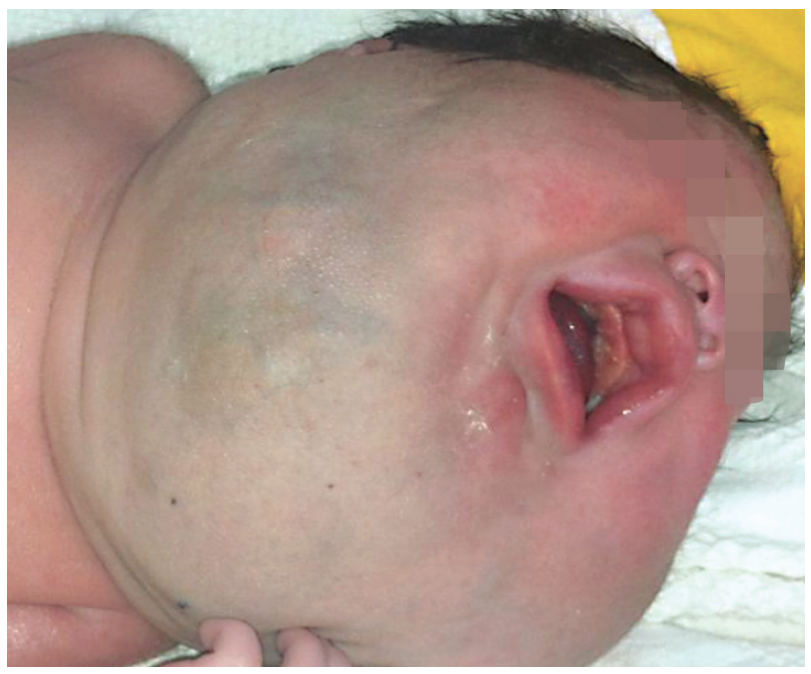

Figura 2. Recién nacido con tumoración cervical en unidad de atención inmediata 
presente caso; ya que estos tumores tienen la propiedad de invadir tejidos subyacentes y producir obstrucción respiratoria alta, como sucedió en nuestro paciente $\mathrm{e}^{7-10}$. Cuando el diagnóstico ecográfico no es concluyente, el uso de resonancia magnética fetal es necesario para evidenciar los vasos sanguíneos afluentes y la extensión de la tumoración ${ }^{11}$.

Durante la evaluación prenatal se debe realizar la medida del líquido amniótico, en donde la presencia de polihidramnios indicaría un patrón obstructivo alto que condiciona compresión esofágica ${ }^{8}$.

El diagnóstico prenatal solo alcanza al $30 \%$ de los casos ${ }^{13}$, los cuales por lo general se desarrollan entre la semana 15 y la 29 de gestación, lo que sugiere un desarrollo tardío de los teratomas dentro del período del embarazo.

La determinación de niveles séricos de alfa- fetoproteína y gonadotropina coriónica es útil en el diagnóstico y seguimiento de este tipo de tumores ${ }^{5}$.

El manejo debe estar orientado a evitar el compromiso por la compresión de la vía aérea la cual puede no ser notoria al nacimiento pero puede progresar rápidamente en las primeras horas de vida ${ }^{12}$; por dicha razón se debe asegurar una ventilación adecuada.

Algunos casos se han manejado con la técnica de tratamiento extra-útero intraparto (EXIT), es decir que durante la cesárea, bajo anestesia general, se extraen la cabeza y los hombros del neonato y de inmediato se procede a intubar al paciente, antes de que este respire. $\mathrm{Si}$ la intubación es fallida se indica traqueostomía. Posteriormente se realiza la atención del recién nacido conforme a los protocolos establecidos ${ }^{12,13}$. Adicionalmente pueden realizarse procedimientos de laringoscopia y broncoscopía para estudio y asegurar la vía aérea.

Por todo lo expuesto se sugiere realizar un monitoreo adecuado del recién nacido a fin de evaluar signos de insuficiencia cardiaca y crecimiento del tumor que condicionaría un patrón obstructivo alto ${ }^{9,11}$.

\section{CONCLUSIÓN}

Los teratomas cervicales congénitos son tumoraciones raras que se incluyen dentro de las alteraciones cervicales en el recién nacido. Del conocimiento de la patología, el diagnóstico oportuno y el manejo adecuado que incluya la aplicación de la estrategia EXIT O traqueotomía oportuna dependerán la evolución a corto y largo plazo, pues aunque es infrecuente siempre hay que saber cómo resolver estos casos de la manera más acertada posible.

\section{REFERENCIAS BIBLIOGRÁFÍCAS}

1. Richard G, Haase G, Applebaum H, Dillon P,Coran A, King $P$, Hodge D. Diagnosis, management, and outcome of cervicofacialteratomas in neonates: A children CancerGroup Study. Journal of Pediatric Surgery (1995)30:2:312-316

2. Kerner B, Flaum E, Mathews H, Carlson D, Pepkowitz S, Hixon H, Graham J. Cervicalteratoma: prenatal diagnosis and long-termfollow up. Prenatal Diagnosis(1998) 18:1: 51-59.

3. Miliaras D, Grimbizis G, Conroy J, Psarra N,Miliaras S, Nowzk N, Bontis J. Novel karyotypicchanges detected by comparative genomichybridization in a case of congenital cervicalinmature teratoma. Birth Defects Research (PartA): Clinical and Molecular Teratology (2005)73:572-576

4. Araujo E, Hélio A, Guimaraes F, Saito M, PiresA. Prenatal diagnosis of a large fetal cervicalteratoma by threedimensional ultrasonography:a case report. Arch Gynecol Obstet(2007) 141-144

5. Viora E, Grassi P, Comoglio F, Bastonero S, Campogrande M. Ultrasonographic detection of fetal cranio-facial hemangioma: case report and review of the literature. Ultrasound Obst Gynecol 2000; 15: 431-34.

6. Shiraishi H, Nakamura M, Ichihachi K, Uchida A, Izumi A, et al. Prenatal MRI in a fetus with a giant neck hemangioma: a case report. Prenat Diagn 2000; 20:1004-7.

7. Rotas M, Haberman S, Merhi Z, Morcos M,. Prenatal Diagnosis of Giant Fetal Truncal Hemangioma by Means of 2- and 3-Dimensional Sonography With Magnetic Resonance Imaging. J Ultrasound Med 2006; 25:527-531

8. Paladini D, Volpe P. Ultrasound of congenital fetal anomalies. 2006. INforma Uk. Pag: 99-101.

9. Kitagawa $H$, Kawase $H$, Wakisaka $M$, Satou $Y$, Saty $H$, Furuta S, Nakada K. Six cases of children with a bening cervical tumor who required tracheostomy. Pediatr Surg Int 2004;20:51-54

10. Tsukimori K, Hojo S, Kawarabayash Yi, Nakanami N, Masumoto N, Kohashi K, Masazumi Tsuneyoshi M. Neck Capillary Hemangioma Associated With Kasabach-Merritt Syndrome. J Ultrasound Med 2007; 26:397-401

11. Yoshida S, Kikuchi A, Naito S, et al. Giant hemangioma of the fetal neck, mimicking a teratoma. J Obstet. Gynaecol 2006;32(1): 47-54.

12. Flores $G$, Reyes M, Dominguez M, Ruiz O. Tumor mixto de células germinales en el cuello asociado a otras malformaciones congénitas en un neonato. Gac Med Méx (2002) Vol 138 No. 6:571-575

13. Miliaras D, Grimbizis G, Conroy J, Psarra N, Miliaras S, Nowzk N, Bontis J. Novel karyotypic changes detected by comparative genomic hybridization in a case of congenital cervical inmature teratoma. Birth Defects Research. Clinical and Molecular Teratology (2005)73:572-576 